\title{
5-Fluorouracil Induces Plasmonic Coupling in Gold Nanospheres: New Generation of Chemotherapeutic Agents
}

\author{
Mona B. Mohamed ${ }^{1,2}$, Nour T. Adbel-Ghani ${ }^{3}$, Ola M. El-Borady ${ }^{2,4 *}$ and Mostafa A. El-Sayed ${ }^{5}$
}

${ }^{1}$ National Institute of Laser Enhanced Sciences (NILES), Cairo University, Giza, Egypt

${ }^{2}$ NanoTech Egypt for Photoelectronics, Dreamland, October city, Egypt

${ }^{3}$ Chemistry Department, Faculty of Science, Cairo University, Giza, Egypt

${ }^{4}$ Chemistry Department, Modern Science \& Arts University, October city, Egypt

${ }^{5}$ Laser dynamics laboratory, Georgia Institute of Technology, Atlanta, USA

\begin{abstract}
Loading 5- Fluorouracil (5-FU) into gold nanoparticles (AuNPs) could enhance its activity as anticancer drug hugely by enhancing its ability for penetration through the cell membrane. Accordingly, this work is devoted to loading 5-FU into AuNPs surface and studying the binding mechanism of the drug to the surface of the gold nanoparticles. Our finding indicates that new absorption band appears at longer wavelength upon loading 5-FU into gold nanospheres capped with citrate. This near IR band is due to induced surface plasmon coupling via hydrogen bonding between 5-FU and surface capping AuNPs. This leads to great enhancement of the drug action as chemotherapeutic as well as photothermal agents. Factors which affect the binding between 5-FU and the AuNPs such as $\mathrm{pH}$, time after mixing the drug with AuNPs, concentration of the 5-FU, have been studied in detail. Accordingly, the binding interaction is proven to be via hydrogen bonding. Upon the investigation of thermal and photo satiability, the formed composite 5-FU@ AuNPs showed high stability towards these factors. The spectral and morphological studies were measured via UV-VIS spectroscopy and Transmission Electron Microscopy (HR-TEM). Remarkable increases in the drug anticancer activity upon loading into AuNPs were observed for the cell viability test of human colon cancer (HCT16).
\end{abstract}

Keywords: Gold nanoparticles; Plasmonic coupling; Cytotoxicity; Colon cancer; 5-Flourouracil

\section{Introduction}

5-Fluorouracil has been in use for about 40 years as a chemotherapeutic agent against cancer. Some of its principal uses are in colorectal cancer, pancreatic cancer as well as inflammatory breast cancer. As a pyrimidine analogue, it is transformed inside the cell into different cytotoxic metabolites which are then incorporated into DNA and RNA, finally inducing cell cycle arrest and apoptosis by inhibiting the cell's ability to synthesize DNA. Some of the adverse reactions of taking 5-fluorouracil might include diarrhoea, nausea, vomiting, stomach/abdominal pain and general weakness $[1,2]$.

Controlled drug-delivery strategies using nanomaterials have made a dramatic impact in medicine that develops highly selective and effective therapeutic and diagnostic modalities [3-5]. There are a number of advantages with nanoparticles in comparison to microparticles. For example, nanoscale particles can travel through the blood stream without sedimentation or blockage of the microvasculature. Small nanoparticles can circulate in the body and penetrate tissues such as tumors. In addition, nanoparticles can be taken up by the cells through natural means such as endocytosis.

Recently, AuNPs have been widely used in cancer therapy and imaging [6], also they have been used as contrast agents in vitro based on their ability to scatter visible light [7]. Au nanorods have been proposed and investigated as a new generation of photothermal agents for cancer therapy and imaging by El-Sayed group [8]. Sokolov et al. [7] used AuNPs conjugated to EGFR antibodies to label cervical biopsies for identification of precancerous lesions.

We believe that loading 5-FU into AuNPs could enhance its activity as anticancer drug hugely due to their ease for biofunctionalization, and penetration through the cell membrane. Accordingly, the aim of this work is to load 5-FU into AuNPs surface and study the nature of binding mechanism between the drug and the surface of AuNPs. Our findings indicate that loading 5-FU upon gold nanospheres capped with citrate induces surface plasmon coupling and cause an assembly for the AuNPs. In the present work, the factors affecting the chemical interaction between the AuNPs and 5-FU such as $\mathrm{pH}$, temperature and reaction time, have been investigated. In addition, the thermal and photostability of the drug loaded into AuNPs have been tested. All the prepared samples were characterized via Transmission Electron Microscopy (TEM) as a primary tool for measuring the particles size, structure and shape, and the plasmonic effect were detected via UVVIS spectroscopy. The anticancer activity against colon carcinoma cells line has been investigated.

\section{Materials and Instruments}

All chemicals were reagent grade and were of highest available purity (A.P.); deionized water was used to prepare all the solutions. All glassware was thoroughly cleaned with aqua regia and rinsed with deionized water prior to use. Hydrogen tetrachloroaurate trihydrate $\left(\mathrm{HAuCl}_{4} \cdot 3 \mathrm{H}_{2} \mathrm{O}-98 \%\right)$ and trisodium citrate $(99.99 \%)$ were purchased from Sigma-Aldrich. 5-Fluorouracil (5FU) was purchased from (Merck, 99\%).

The UV-VIS absorption spectra were recorded on a PERKIN-

${ }^{*}$ Corresponding author: Dr. Ola Mohamed El Borady, Chemistry Department Modern Science \& Arts University, October city, Egypt, Tel: 01004725266; E-mail: olachem_elborady@yahoo.com

Received September 08, 2012; Accepted September 10, 2012; Published September 12, 2012

Citation: Mohamed MB, Adbel-Ghani NT, El-Borady OM, El-Sayed MA (2012) 5-Fluorouracil Induces Plasmonic Coupling in Gold Nanospheres: New Generation of Chemotherapeutic Agents. J Nanomed Nanotechol 3:146. doi:10.4172/2157 7439.1000146

Copyright: (c) 2012 Mohamed MB, et al. This is an open-access article distributed under the terms of the Creative Commons Attribution License, which permits unrestricted use, distribution, and reproduction in any medium, provided the original author and source are credited. 
ELEMER LAMBDA 40 spectrophotometer using $1 \mathrm{~cm}$ matched quartz cells. The transmission electron microscopy (HR-TEM) images were carried out in Nanotech Company for photo-electronic, Dreamland, 6-October, Egypt. The HR-TEM is JOEL JEM-2100 operating at 200 $\mathrm{kV}$ equipped with Gatan digital camera Erlangshen ES500. The $\mathrm{pH}$ measurements were adjusted by using JENWAY $4330 \mathrm{pH}$ meter. The cell viability was recorded via ELISA reader (BIOTEK, Model: ELX 800) wavelength range: 400 to $750 \mathrm{~nm}$ with four built in filters. The particle size for the 5-FU@AuNPs was measured on Zetasizer, Malvern, UK, Model: Zetasizer nano series (Nano ZS), Size range $(\mathrm{nm}): 0.6: 6000 \mathrm{~nm}$.

\section{Experimental}

Preparation of Spherical AuNPs and 5-FU@AuNPs: Au nanoparticles were prepared by the citrate reduction of $\mathrm{HAuCl}_{4} \cdot 3 \mathrm{H}_{2} \mathrm{O}$ as described previously [9]. Tri-sodium citrate $(0.0388 \mathrm{M}, 10 \mathrm{~mL})$ was added quickly to a boiling $\mathrm{HAuCl}_{4}$ solution $\left(1 \times 10^{-3} \mathrm{M}, 100 \mathrm{~mL}\right)$. Strong pink color appears as an indication of the formation of AuNPs. The absorption spectrum of the obtained sample was measured via UV-VIS spectrophotometer, and the particle shape and size were characterized using TEM.

5-FU@AuNPs were prepared by adding an aqueous solution of $(0.01 \mathrm{M}, 0.0650 \mathrm{gm}) 5$-fluorouracil to $50 \mathrm{~mL}$ of the synthesized gold nanoparticles and stirred effectively under ultra-sonication to enhance the interaction between 5-fluorouracil and gold nanoparticles. The obtained composite has been characterized via absorption spectroscopy and HR-TEM.

\section{Cytotoxicity and cellular uptake for the 5-Fluorouracil, AuNPs and 5-FU@AuNPs}

Cytotoxicity of 5-Fluorouracil, AuNPs and 5-FU@AuNPs was assessed by cell culture method using established cell line derived from human colon cancer (HCT16).

Cell culture: HCT16 established cell line was commercially obtained from The Holding Company for Biological Products \& Vaccines "VACSERA", Cairo, Egypt as a monolayer sheet in $20 \mathrm{ml}$ of RPMI growth media supplemented with $10 \%$ fetal calf serum (Biowest, Germany). The cells were routinely propagated and maintained as well as the cells viability and plate preparation was carried out in Virology \& Immunology Unit, National Cancer Institute, Cairo University, Egypt.

Cells viability and plate preparation: Cell viability and cell number were determined after staining of cells with trypan blue exclusion test (Biowest USA). Sterile 24 wells tissue culture plate was seeded with $1 \times 10^{5}$ cells $/ \mathrm{ml}$ and then was incubated at $37^{\circ} \mathrm{C}$ in $5 \% \mathrm{CO}_{2}$ incubator and left for $24 \mathrm{hrs}$ to allow formation of confluent monolayer. After $24 \mathrm{hrs}$, the media was replaced with three different concentrations of 5-Fluorouracil $\left(1 \times 10^{-4}, 1 \times 10^{-5}\right.$ and $\left.1 \times 10^{-6} \mathrm{M}\right)$, AuNPs $\left(1 \times 10^{-4}, 1 \times 10^{-5}\right.$ and $\left.1 \times 10^{-6} \mathrm{M}\right)$, and of composite 5-FU@AuNPs $\left(1 \times 10^{-4}, 1 \times 10^{-5}\right.$ and $1 \times 10^{-6}$

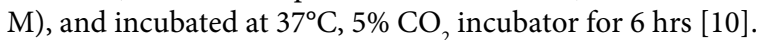

Cytotoxicity assay: It was estimated by using neutral red colorimetric assay performed according to Babichand and Borenfreund methodology [10]. After $6 \mathrm{hrs}, 100 \mu \mathrm{l}$ of neutral red dye (Biochrom L6313; $0.003 \%$ in $0.9 \% \mathrm{NaCl} /$ medium with $2 \%$ (FBS) Fetal Bovine Serum) was added to the plate and incubated for $3 \mathrm{hrs}$ at $37^{\circ} \mathrm{C}$. Then, the plate was washed with $200 \mu \mathrm{l}$ of Phosphate Buffer Saline (PBS) and the cells were lysed with $200 \mu$ of lysis buffer and placed on shaker for $20 \mathrm{~min}$ to extract dye from the viable cells. The extracted dye was then measured using ELISA reader. The relative percent of cell viability was calculated by $[\mathrm{A}]$ test/ $[\mathrm{A}]$ control $\mathrm{x} 100$, where $[\mathrm{A}]$ test is the absorbance of the test sample and $[\mathrm{A}]$ control is the absorbance of control sample. The cell control represents $100 \%$ viability.

\section{Results and Discussion}

The visible absorption spectra of the wine red colloidal AuNPs showed a well-defined absorption band with $\lambda_{\max }=522 \mathrm{~nm}$ (Figure 2). This value is a characteristic for Surface Plasmon Resonance (SPR) absorbance for spherical AuNPs [11]. The wavelength was not significantly modified, during a year after preparation, thus suggesting the stability of the colloidal solution. The size and shape of the gold colloid particles have been measured by TEM imaging. A representative TEM image of these particles is given in Figure 2. The particles are mostly spherical. From the sizes of a great number of particles, measured on the TEM images, an average size (diameter) of the synthesized nanoparticles is $12-14 \mathrm{~nm}$ (histogram Figure 2).

Upon loading the 5-FU into AuNPs capped with sodium citrate, a gradual color change from red to purple then blue has been observed and a new band starts to appear at higher wavelength $\sim 690 \mathrm{~nm}$. The $\lambda_{\max }$ of this new absorption band continues its shift towards higher wavelengths with remarkable increase in intensity with time Figure 3B. The increase of absorbance for the new band at $690 \mathrm{~nm}$ was accompanied by a decrease in intensity of the SPR band of spherical AuNPs at $522 \mathrm{~nm}$ and a slightly shift was observed in its $\lambda_{\max }$ from $522 \mathrm{~nm}$ to $530 \mathrm{~nm}$ Figure 3A. This shift is due to the change in the dielectric constant of the solution [12]. The color change, as an effect of agglomeration (assembly of the particles), is a well-understood phenomenon [13]. When the interparticle distance in the assembly decreases to less than about the average particle diameter, the electric dipole-dipole interaction and coupling between the plasmons of neighboring particles in the assembly has resulted in the bathochromic shift of the absorption band which is called plasmon coupling band [14]. The diameter of the agglomerated particles was measured by using zetasizer analysis and most of them have $\sim 48 \mathrm{~nm}$, see supplemental information.

Increasing the concentration of 5-FU has a significant effect on the degree of the assembly and the nanocrystals agglomeration kinetics. As shown in Figure 4, an increase in the concentration of the drug leads to further agglomeration (assembly) of the gold nanocrystals.

We can assume that 5-FU bind to the citrate coating the AuNPs surface, inducing particles agglomeration. The interaction occurred through the formation of two hydrogen bonds; one between $\mathrm{COO}^{-}$of citrate and the $\mathrm{NH}$ of the drug 5-FU and the other between carbonyl group in the drug and $\mathrm{OH}$ in sodium citrate as shown in Figure 5.

The degree of plasmonic coupling and AuNPs assembly coated by $5-\mathrm{FU}$ were also found to be tunable by $\mathrm{pH}$, where the $\mathrm{pH}$ of the medium is a vital factor that determines the stability of the composite.<smiles>O=c1cc(F)[nH]c(=O)[nH]1</smiles>

$\mathrm{H}$

Figure 1: The chemical structure of 5-flourouracil. 

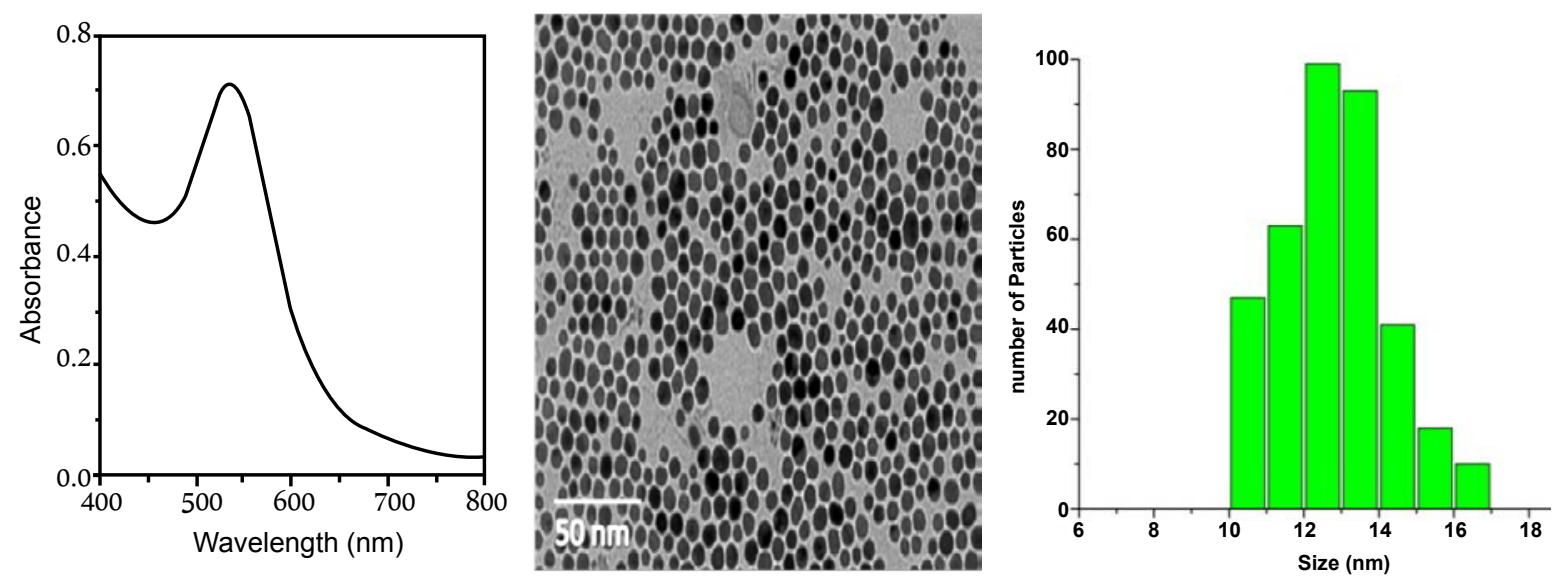

Figure 2: The UV-Vis spectrum of Au nanoparticles and transmission electron micrographic image of gold nanoparticles and their histogram of size distribution.



Figure 3: A)The time-dependent UV-Vis spectra of 5-FU@AuNPs: (a) pure AuNPs, (b-f) after mixing with 5-FU, b) 10 mins, c) 30 mins, d) 2 hours, e) 4 hours, f) 6 hours, B) Time dependence for the shift of $\lambda_{\max }$ of the plasmon coupling band for 5-FU@AuNPs.

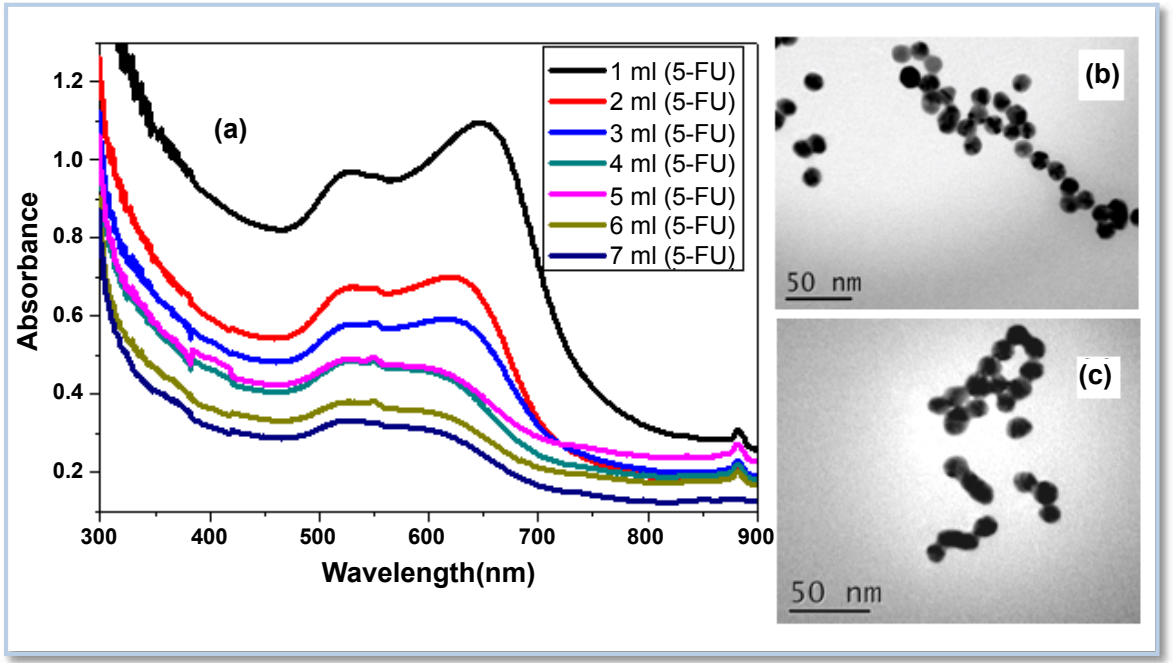

Figure 4: (a)UV-Vis spectra of 5-FU @AuNPs formed with different volume ratio of 5-FU, (b) TEM images for $1 \mathrm{~mL}: 0.5 \mathrm{~mL}$ of Au:5-FU show mostly linearly arranged assemblies of NPs, (c) TEM images for $1 \mathrm{~mL}: 1 \mathrm{~mL}$ of Au:5-FU, while the nanoparticles assemblies are more complex. 
For this reason, the 5-FU has been loaded into gold capped citrate nanocrystals at different $\mathrm{pH}$ values using $0.1 \mathrm{M} \mathrm{NaOH}$ and $0.1 \mathrm{M} \mathrm{HCl}$. The absorption spectra of 5-FU @AuNPs at different $\mathrm{pH}$ values have been monitored (Figure 6) and the resultant composites have been characterized via HR-TEM (Figure 7).

Assembly of AuNPs can be produced by the surface acid-base reactions of the individual gold nanoparticles. Recent work of Kleinermanns et al. [16], reported the ensemble of AuNPs [15] modified with an ionizable and $\mathrm{pH}$-sensitive monolayer of Thiobarbituric Acid (TBA). It was found that nanoparticle assembly can be produced in a controlled fashion by the variation of the $\mathrm{pH}$ of the solution [16]. Our results show that the interparticle spacing in Au assembly mediated by anticancer drug 5-FU was also found to be tunable by $\mathrm{pH}$ as shown with as TBA. One notable feature in Figure 6 is that, the plasmon coupling band of the assembled AuNPs at $\sim 690 \mathrm{~nm}$ appeared gradually for the solutions of $\mathrm{pH} 3$ to 6 with a color change from wine red to purple then blue with increase $\mathrm{pH}$. This may be assigned to lowering of the $\mathrm{pH}$ of solution leads to protonation of the amine group in the drug $(\mathrm{N}=1$, Figure 1), allowing hydrogen bond interactions between the drug and carboxylic group in the citrate inions that capping gold nanoparticles, thus leading to assembly of AuNPs. On the other hand, the absorption spectra of the solutions with high $\mathrm{pH}$ values (from $\mathrm{pH}=7$ to $\mathrm{pH}=11$ ), revealed no bands rather than the SPR band of AuNPs Figure 6(b).

A successive shift in SPR of AuNPs from 520 to $531 \mathrm{~nm}$ along with reduction in intensity was observed upon the addition of 5-FU for the solutions of $\mathrm{pH}$ from 2.0 to 11.0

All of these observations were also evident from TEM studies as shown in Figure 7. The pH-dependent in optical properties has implications for controlled release of molecules from the nanoparticle assembly, an area of importance in controlled drug release.

If gold nanoparticles are coated with a drug shell, the drug can be released from the carriers either via enzymatic activity or changes in physiological conditions such as $\mathrm{pH}$, osmolality, or temperature [17] to be taken up by tumor cells. For this reason the thermal stability of 5-FU@AuNPs was investigated, by heating a solution of 5-FU@AuNPs to various temperature degrees in the range of $25-100^{\circ} \mathrm{C}$. Furthermore, this study gives an impression to the decomposition temperature of the composite briefly. With increasing the temperature up to $90^{\circ} \mathrm{C}$ the electronic absorption spectrum Figure 8(a) shows no significant change in the absorption maximum of the two characteristic bands, the SPR band of AuNPs at $522 \mathrm{~nm}$ and the band due to plasmonic coupling

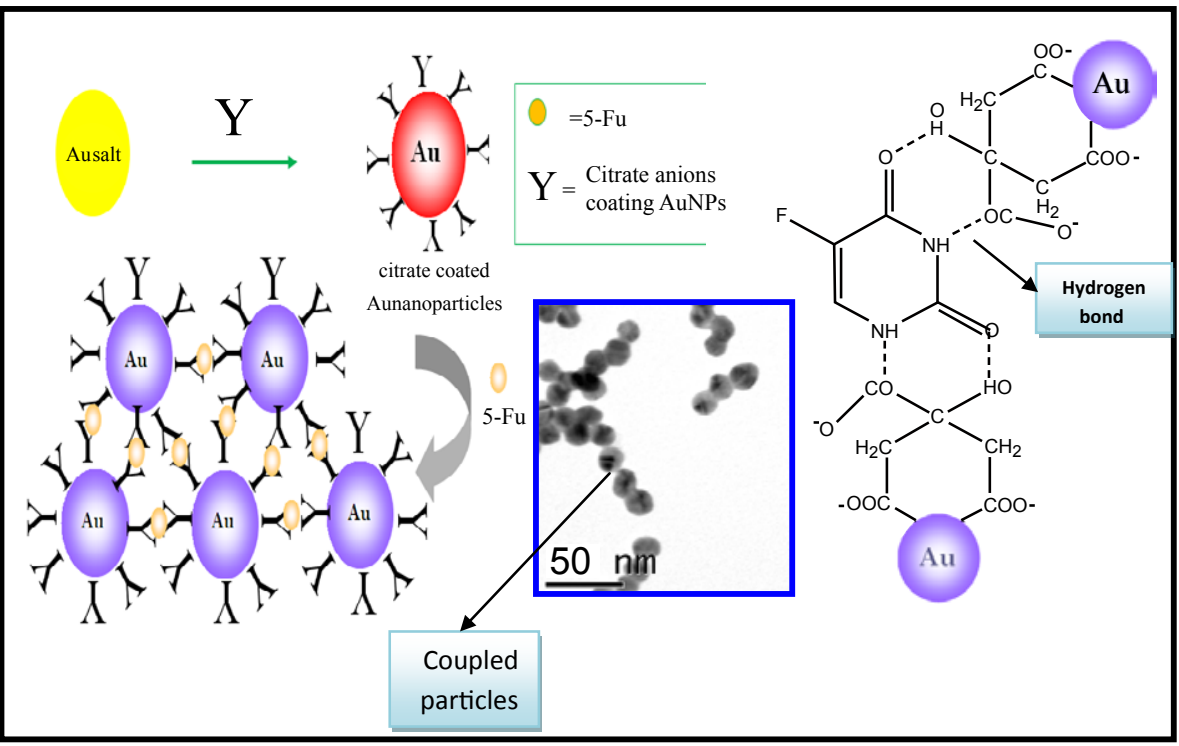

Figure 5: The chemical formula postulated for the linkage between 5-FU and AuNPs, and its TEM image of their assembly.
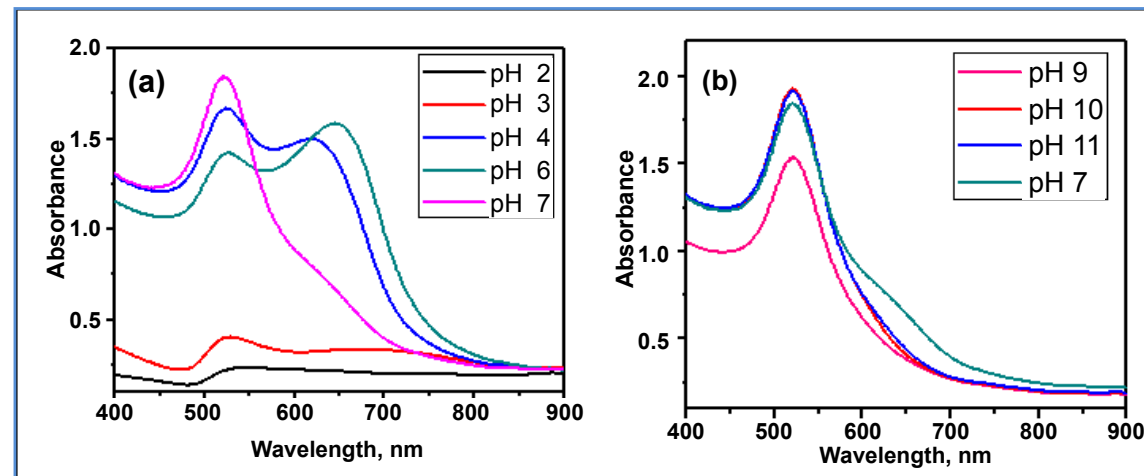

Figure 6: The UV-Vis spectra of 5-FU@AuNPs at different $\mathrm{pH}$ values: (a) acidic media $\mathrm{pH}=2-7$, (b): basic media $\mathrm{pH}=7-11$. 


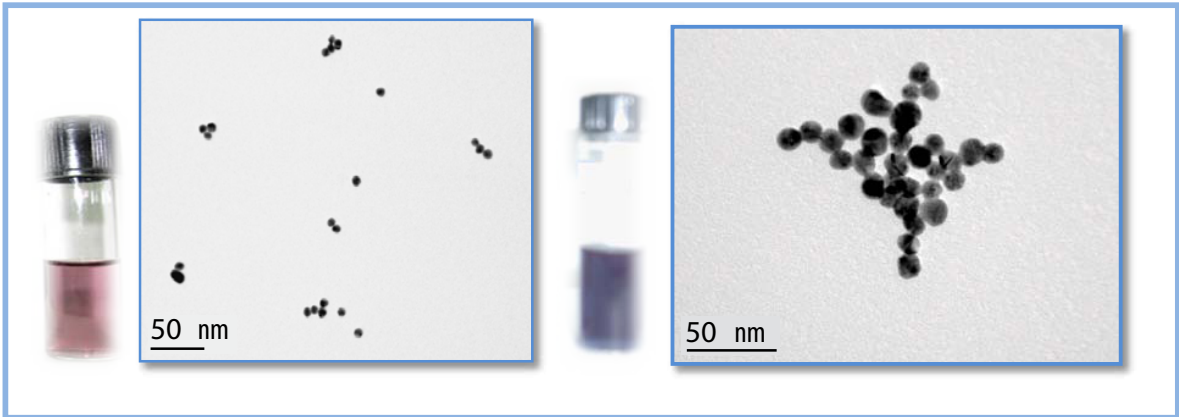

Figure 7: (a) The TEM images of 5-FU@AuNPs at $\mathrm{pH}=3$ with the purple color showing the beginning of the assembly with two or three particles (b): at pH=6, while the assembly is more complex.

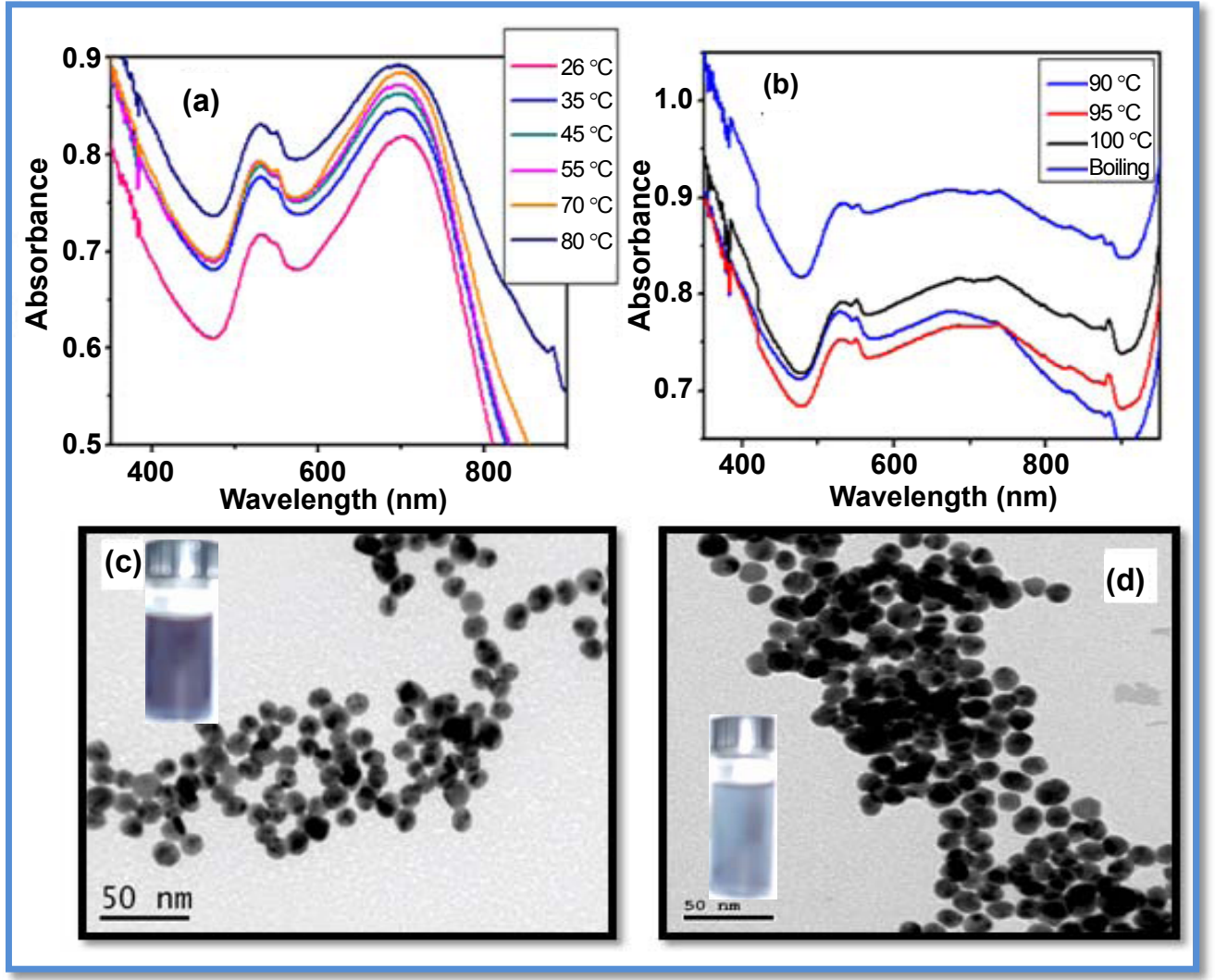

Figure 8: Effect of high temperature on absorption spectra of an aqueous solution of 5-FU@ AuNPs :(a) from $25-80^{\circ} \mathrm{C}$ (b) $90-100^{\circ} \mathrm{C}$, (c) TEM image and photo at $25^{\circ} \mathrm{C}$ (d) TEM image and photo after boiling.

for the composite at $695 \mathrm{~nm}$, which indicates the high stability of the formed composite up to this temperature.

Beyond $90^{\circ} \mathrm{C}$, both of the SPR absorption band and plasmon coupling band of 5-FU@AuNPs nanocomposite are merged together Figure 8(b). This observation suggests that the micelles encapsulating the gold nanoparticles are relatively destroyed, and a black precipitate of aggregated gold powder is formed. This leads to the possibility that the decomposition temperature of the most stable micelles used in this work is in the range of $90-100^{\circ} \mathrm{C}$.

The effect of UV-light on the 5-FU@AuNPs composite was observed using absorption spectrophotometer. To demonstrate the irradiation effect of UV-light on the sample solution, $5 \mathrm{ml}$ colloidal solution of the
5-FU@AuNPs were put in $5 \mathrm{~cm}$ quartz cell under the UV-light source $(\lambda=254 / 365 \mathrm{~nm})$ and exposed to different irradiation time.

The results obtained from absorption show that 5-FU@AuNPs before irradiation to light has two $\lambda_{\max }$ one at $522 \mathrm{~nm}$ due to the SPR of AuNPs and another band at $695 \mathrm{~nm}$ due to plasmon coupling of NPs. By irradiation, the 5-FU@AuNPs as a function of time and the optical density shows slight decrease during the first hour without any remarkable shift in the corresponding wavelengths of each band, Figure 9. This may be attributed to the capping strength of 5-FU which form a composite with AuNPs preventing the nanoparticles from aggregation during the irradiation time. On increasing the time of irradiation for 3 hours, a remarkable decrease in the intensity of both bands without 


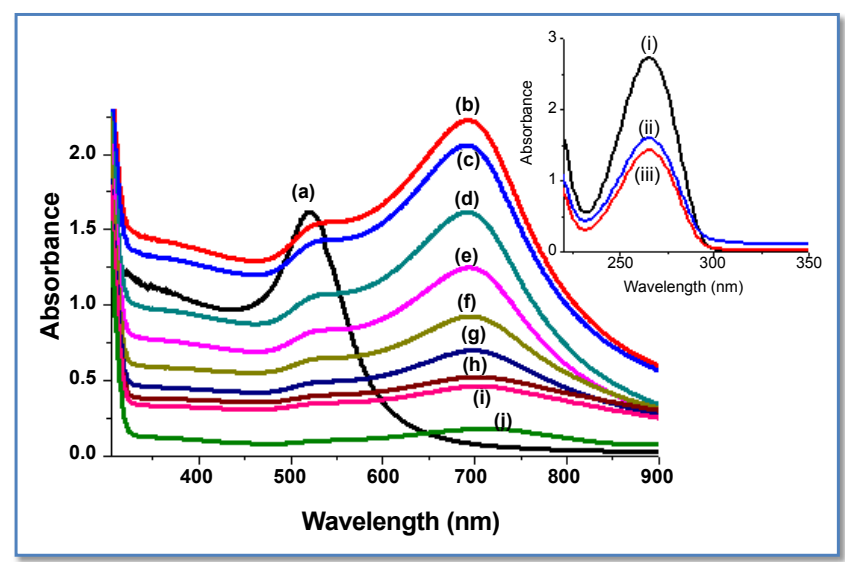

Figure 9: shows the effect of UV-light on AuNPs@5-FU: (a) AuNPs, (b) 5-FU@AuNPs without irradiation(c-j) after irradiation with time interval 1 hour until 6 hours. The inset figure (i) 5-FU (ii) 5-FU@AuNPs without irradiation and (iii) irradiated 5-FU@AuNPs.

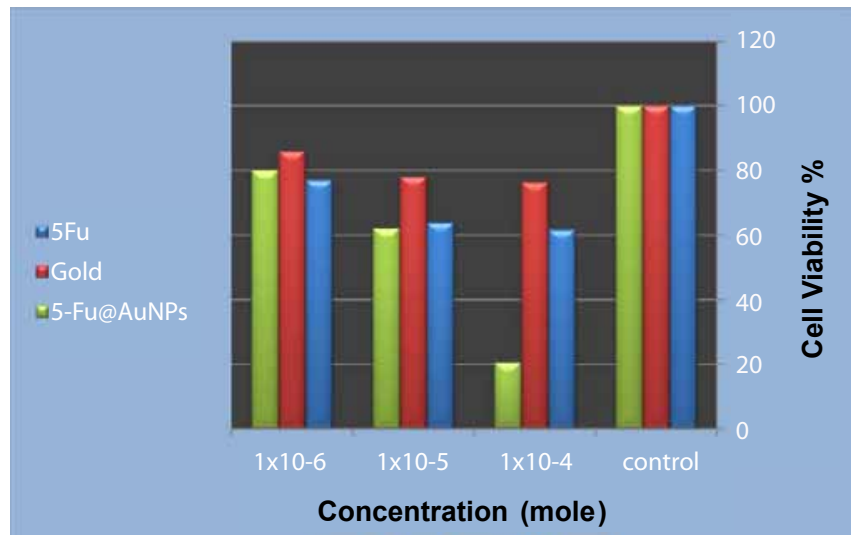

Figure 10: The percentage viability of cells at different concentrations of 5-Fu, AuNPs, and 5-FU@AuNPs, respectively.

shift in the corresponding wavelengths was observed. The bands continue decaying as the time increase up to 6 hours of irradiation. This may be due to the regular release of the drug from the surface of AuNPs with the decomposition of the gold nanoparticles combined with an aggregation in the solution. The results suggest that the use of these particles in magnetic fluid hyperthermia with the exposure to UV radiation will be safe and the particles will do well up to 3 hours.

During the time of exposure, the $n-\pi^{*}$ transition band of the 5-FU molecule showed no changes as observed in the inset Figure 9.

\section{Cytotoxicity test}

In vitro cell viability (Cytotoxicity studies): Neutral red assay using the HCT16 (colon carcinoma cells line) was performed to analyze the potential toxicity of the AuNPs, 5-FU and 5-FU@AuNPs, in concentration $\left(1 \times 10^{-4}, 1 \times 10^{-5}\right.$ and $\left.1 \times 10^{-6} \mathrm{M}\right)$, After incubation time of 6 hours, viable cells have the ability to absorb neutral red whose absorption could be estimated at $570 \mathrm{~nm}$; the value at which it is correlated to the number of living cells (Figure 10). It was found that at low concentration, $1 \times 10^{-5}$ and $1 \times 10^{-6} \mathrm{M}$ of 5-FU and 5-FU@ AuNPs nanocomposites show low cell viability while at slightly high concentration 1x10-4 M of 5-FU@AuNPs shows high cell viability (cells death) about 20\% compared with AuNPs and 5-FU which indicates the effectiveness for cell toxicity of this nano composites in cancer treatment and open the door for using anticancer drug loaded over nanoparticles. On the other hand, citrate stabilized AuNPs were shown to have little effect on this particular cell line as described by Connor et al. [18], and hence, we have used them for proper comparison. The results suggest that the 5-FU@AuNPs nanocomposites show excellent dispensability and long-term stability in physiological solution viability from $20-80 \%$ has been demonstrated compared to the control. Thus, on the basis of the in vitro data, the 5-FU@AuNPs nanocomposites are good potential candidates as a drug delivery for further in vitro studies as well as in vivo applications.

\section{Conclusion and Future Perspective}

The nature of binding the anticancer drug 5-FU with the prepared AuNPs has been established using UV-Vis spectroscopy and TEM imaging. The results revealed 5-FU bind to the citrate coating the AuNPs surface, inducing particles agglomeration i.e. assembly of the NPs, which increase gradually with time. The interaction occurred through the formation of two hydrogen bonds one between $\mathrm{COO}^{-}$of citrate and the $\mathrm{NH}$ of the drug 5-FU and the other between carbonyl group in the drug and $\mathrm{OH}$ in citrate. The previous discussion also referred to the formation of 5-FU@AuNPs in acidic media due to protonation in 5-FU molecule that enhance interaction with citrate capped AuNPs. Furthermore, this investigation shows high thermal and photo stability for the 5-FU@AuNPs.

We have found substantial enhancement of the anti cancer effect of 5-FU@AuNPs against HCT16 (colon carcinoma cells line) compared to the same drug in typically administered free form or as prepared AuNPs only. The improvement was attributed to enhanced intracellular transport followed by the subsequent release in lysosomes. Enhanced activity and nanoparticle carriers will make possible the reduction of the overall concentration of the drug, renal clearance and side effects.

\section{Acknowledgment}

The authors thank Ola El-Sayed for her great efforts in preparing and measuring the cell viability for the materials under study in her lab "Virology \& Immunology Unit, National Cancer Institute, Cairo University, Egypt".

\section{Financial \& competing interest disclosure}

This study was supported financially by grant from Cairo University titled "Nanomedicine: New Targeting Approach to Decrease the Side Effects of Chemotherapy ". The authors have no other relevant affiliations or financial involvement with any organization or entity with a financial interest in or financial conflict with the subject matter or materials discussed in the manuscript apart from those disclosed. No writing assistant was utilized in the production of this manuscript.

\section{References}

1. Oguri T, Bessho Y, Achiwa H, Ozasa H, Maeno K, et al. (2007) MRP8/ABCC11 directly confers resistance to 5-fluorouracil. Mol Cancer Ther 6: 122-127.

2. Seo E, Abei M, Wakayama M, Fukuda K, Ugai H, et al. (2005) Effective gene therapy of biliary tract cancers by a conditionally replicative adenovirus expressing uracil phosphoribosyltransferase: significance of timing of 5-fluorouracil administration. Cancer Res 65: 546-552.

3. Sibnath K, Raju VR (2010) Anti-Cancer drug loaded iron-gold core-shell nanoparticles (Fe@Au) for magnetic drug targeting. J Nanosci Nanotechno 10: 1-13.

4. McCarthy JR, Perez JM, Bruckner C, Weissleder R (2005) Polymeric nanoparticle preparation that eradicates tumors. Nano Lett 5: 2552-2556.

5. Chigumbu N, lyuke S, Pillay V, Sehliselo Ndlovu (2012) In Vitro evaluation of the physicochemical effects of drug loaded carbon nanotubes on toxicity. $J$ Nanomed Nanotechnol 3: 1-7.

6. Ferrari M (2005) Cancer nanotechnology: opportunities and challenges. Nat Rev Cancer 5: 161-171. 
Citation: Mohamed MB, Adbel-Ghani NT, El-Borady OM, El-Sayed MA (2012) 5-Fluorouracil Induces Plasmonic Coupling in Gold Nanospheres: New Generation of Chemotherapeutic Agents. J Nanomed Nanotechol 3:146. doi:10.4172/2157-7439.1000146

7. Sokolov K, Follen M, Aaron J, Pavlova I, Malpica A, et al. (2003) Real-time vital optical imaging of precancer using anti-epidermal growth factor receptor antibodies conjugated to gold nanoparticles. Cancer Res 63: 1999-2004.

8. Huang X, El-Sayed IH, Qian W, El-Sayed MA (2006) Cancer cell imaging and photothermal therapy in the near-infrared region by using gold nanorods. J Am Chem Soc 128: 2115-2120

9. Liu J, Lu Y (2006) Preparation of aptamer-linked gold nanoparticle purple aggregates for colorimetric sensing of analytes. Nat Protoc 1: 246-252.

10. Podsiadlo P, Sinani VA, Bahng JH, Kam NW, Lee J, et al. (2008) Gold nanoparticles enhance the anti-leukemia action of a 6-mercaptopurine chemotherapeutic agent. Langmuir 24: 568-574.

11. Handley DA (1989) Methods for synthesis of colloidal gold: Colloidal Gold Principles, Methods, and Applications. Academic Press, San Diego.

12. Mann S, Shenton W, Li M, Connolly S, Fitzmaurice D (2000) Biologically programmed nanoparticle assembly. Adv Mater 12: 147-150.
13. Mayya KS, Patil V, Sastry M (1997) On the stability of carboxylic acid derivatized gold colloidal particles: the role of colloidal solution $\mathrm{pH}$ studied by optical absorption spectroscopy. Langmuir 13: 3944-3947.

14. Mirkin CA, Letsinger RL, Mucic RC, Storhoff JJ (1996) A DNA-based method for rationally assembling nanoparticles into macroscopic materials. Nature 382 : 607-609.

15. Turkevich J, Stevenson PC, Hillier J, Discuss J (1951) A study of the nucleation and growth processes in the synthesis of colloidal gold. Faraday Soc 11: 55-75.

16. Peng Z Walther T, Kleinermanns K (2005) Influence of intense pulsed lase irradiation on optical and morphological properties of gold nanoparticle aggregates produced by surface acid-base reaction. Langmuir 21: 4249-4253.

17. Alexiou C, Arnold W, Klein RJ, Parak FG, Hulin P, et al. (2000) Locoregiona cancer treatment with magnetic drug targeting. Cancer Res 60: 6641-6648.

18. Connor EE, Mwamuka J, Gole A, Murphy CJ, Wyatt MD (2005) Gold nanoparticles are taken up by human cells but do not cause acute cytotoxicity. Small 1: 325- 327 